\title{
Clinical characteristics and outcome determinants of stroke rehabilitation
}

\author{
Ravi P Ruberu ${ }^{1}$, Shaad Manchand ${ }^{1}$, Patrick Conolly ${ }^{1}$, Laura Gilgan ${ }^{1}$, Deepal Lecamwasam ${ }^{1}$ \\ Journal of the Ceylon College of Physicians, 2012, 43, 28-31
}

\begin{abstract}
Introduction: Heterogeneity of stroke outcome measures has increased the complexity of there usefulness across institutions. We sought to identify factors that determine post stroke functional outcome and discharge destination following rehabilitation.
\end{abstract}

Method: Retrospective study of 482 stroke patients admitted to a rehabilitation centre between July 07 to July 12 were studied. Selection criteria included ischemic or hemorrhagic stroke with completed admission notes. Sixty-eight variables were analysed. Partition modeling was used to identify significant variables.

Results: Total of 426 patients met inclusion criteria (mean age 70.67 SD $=13.31$, SEM 0.645 ; male $58 \%, n=245$ ). Mean length of rehabilitation was $48.36 \mathrm{~d}$. Majority of patients were from home prior to stroke and $80 \%$ returned home, the mean length of rehabilitation was 45.22 d. A significant difference was noted in the total Functional Independence Measure (FIM) for patients who were discharged home compared to those discharged to high level residential care $(\mathrm{HLOC})(\mathrm{p}<0.001)$ or low level residential care (LLOC) $(p=0.0497)$, and those who were discharged to LLOC compared to HLOC ( $p=0.0044$ ). Total FIM $\geq 77$, carer support, and age $\leq 77$ y were associated with returning home. For patients with total FIMS 36-77, age $<82$ y and carer support were associated with returning home. HLOC was predictable if age $\geq 82 \mathrm{y}$, length of stay in acute hospital $\geq 14 \mathrm{~d}$, and FIM for upper body dressing of $<5$.

Conclusion: Total FIMS together with other predictors prior to enrolling in inpatient stroke rehabilitation would improve efficiency and patient outcomes.

\footnotetext{
${ }^{1}$ Royal Adelaide Hospital, North Terrace, Adelaide, SA 5000, Australia.
}

\section{Background}

Impact of stroke to both personal life and its socioeconomical environment is substantial, and assumes a growing impact globally on media attention, patient and carer knowledge, service development and research ${ }^{1}$. Rapidly growing elderly population has called for robust data collection to meet the demands of economic and health infrastructure globally. Unfortunately even at the end of the 20th century, international community lack consistency and stringency in quantifying health problems ${ }^{2}$ leading to gaps in knowledge that effect planning. Data from 1990s showed cerebrovascular disease as a leading cause of death ${ }^{3}$. There has been a steady rise in mortality over the following decade accounting for 12.9 million deaths collectively due to ischemic heart disease and stroke in 2010 world wide i.e. 1 in 4 in comparison to 1 in 5 in 1990s ${ }^{4}$. In 2003 around 346,700 Australians had had a stroke at some time in their lives. During this period 282,600 with stroke had a disability and in over $50 \%$, the disability was a direct event from stroke ${ }^{5}$. $1.8 \%$ of total health system expenditure (AUD \$ 896 million) was allocated to cerebrovascular disease, where aged care homes accounted for half of the funds and $40 \%$ for inpatient care. In Australia as in most other countries funding for stroke rehabilitation including other fields of inpatient care, is based on 'case mix' - referring to the number and types of patients or diseases being treated by a health care provider. This system has undergone several revisions as funding on an existing budget, carried over from one year to the next with slight adjustment for productivity and cost increases has little incentive to increase productivity. In the early 1990s, a Functional Independence Measure (FIM) of the functionally related groups was initiated in the USA to facilitate service productivity by implementing newer more effective treatment ${ }^{6}$. There is little consistency across Australia in their use of utility assessment scores. A recent study showed over 300 assessment tools were in use across Australia ${ }^{7}$. Among several others, FIM score showed high consistency. We have used FIM scores in our study as it was the most consistent scoring system which was readily accessible that had demonstrated adequate levels of inter-rater and intra-rater reliability and has been validated as a reliable outcome measure of stroke recovery. 
Outcome prediction including length of stay following stroke are usually carried out during multidisciplinary meetings based on individual therapist experience and their interaction with patients. While this being an important matter to both the patient/family and staff/institution alike, prediction outcome based on experience alone has poor consistency and reliability. This study undertook the task of developing early stroke rehabilitation prediction criteria.

\section{Methodology}

A retrospective study of 482 stroke patients admitted to a rehabilitation centre from July 07 to July 12 was done. Selection criteria included ischemic or hemorrhagic stroke with completed admission notes. Sixty-eight variables including patients' demography, clinical details, individual FIM components (admission and discharge) were analysed using JMP 10 (SAS group) statistical software. Partition modeling was used to identify variables with highest likely hood. Student t-test was carried out to assess the level of significance between total admission FIM and discharge destinations.

\section{Results}

Of 482 stroke rehabilitation patients 426 patients met inclusion criteria (mean age 70.67 SD $=13.31$, SEM 0.645 ; male $58 \%, n=245$ ). Mean length of rehabilitation was $48.36 \mathrm{~d}$. Majority $(98 \%, \mathrm{n}=419)$ of patients were from home prior to stroke and $80 \%$ $(n=333)$ returned home. For those who returned home the mean length of rehabilitation was $45.22 \mathrm{~d}$ where $66 \%(n=221)$ were discharged within 50 days (Table 1). Student $t$ test for total admission functional independence measure score (FIMS) by discharge destination was performed. A significant difference in the total FIMS was seen for patients who were discharged home compared to those discharged to either high level residential care $(H L O C)(p<0.001)$ or low level residential care (LLOC) $(p=0.0497)$. A significant difference was also found between those who were discharged to LLOC compared to HLOC $(p=0.0044)$. Total FIMS of 77 or more, carer support, and age less than $77 \mathrm{y}$ were associated with returning home compared to patients with total FIMS 36-77, age $<82 \mathrm{y}$ and carer support. HLOC was predictable in those who were $>=82 \mathrm{y}$, length of stay in acute hospital $>=14 \mathrm{~d}$, FIMS for upper body dressing of $<5$.

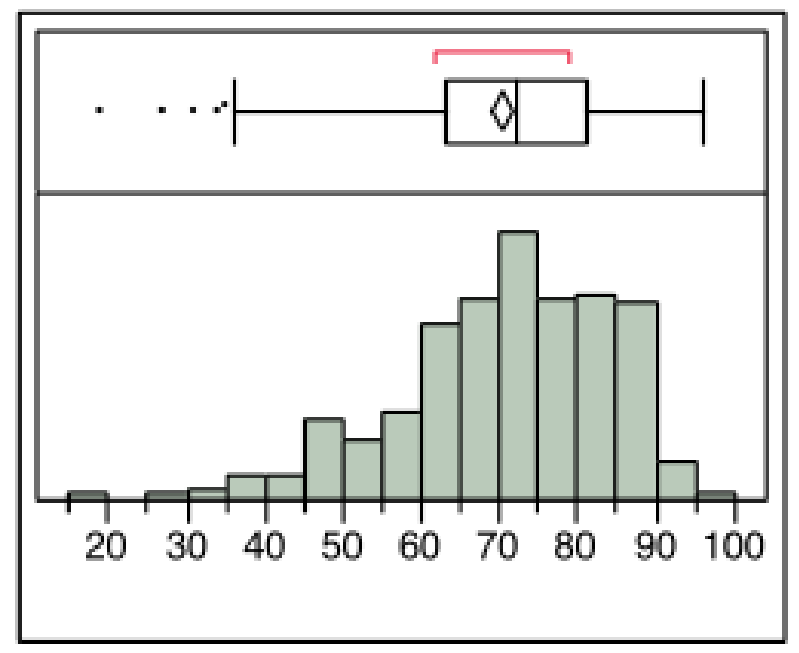

Figure 1. Whisker and box plot along side the histogram showing age distribution of study population ( $x$ axis, age in years). Mean age of 70.7 years (SD 13.31).

\section{Table 1. Descriptive summary of study population}

Total Number

Total Number included

Mean Age

Rehabilitation period (mean)

Premorbid admission status

Discharge destination
482

426 (56 excluded)

70.67 y (SD 13.31;

SM 0.745), Males

245 Females 181

48.36 days

$98 \%$ from home

$80 \%$ returned home (rehabilitation time 45.22 days; $66 \%$ were discharged within 50 days);

\section{Discussion}

Purpose of this study was to identify key variables related to the patient at the time of admission to stroke rehabilitation ward that could be used to recognize patient's discharge destination. We believe our research outcome will contribute to improving the efficiency of appropriate care as well as developing cost effective schemes that is individualized to the 
patient. In the following discussion we aim to explore our findings in light of current patient demographics in Australia, quality of similar work by others, and future direction of stroke rehabilitation research.

Department of Health and Ageing of the Australian Government funded the development of clinical guidelines for acute stroke management and stroke rehabilitation and recovery as part of the National Stroke Unit Program. These guidelines are helpful in providing a high standard of evidence-based care across the nation. Whilst there is an abundance of literature supporting dedicated stroke units to provide greater adherence to processes of care and improved mortality as well as greater degree of independence following discharge ${ }^{8}$ clinical predictive factors of stroke outcome have been less well studied. Comparison of 33 studies by Jongbloed showed that only three studies had over 400 cases analysed. These were significantly limited in their selection of variables; there were no detail analysis of functional scores. Only two studies reported on patients between $70-80$ y and in all other studies the cohort of patients were below $70 \mathrm{y}$ of age. There were wide variety of attributes used as functional status and for the purpose of comparison, function was defined as the ability to perform activities of daily living ${ }^{9}$. We recognize the hazard of using functional markers only at discharge and total scores, not individual elements of a scoring system. Firstly, the practical validity of this system for therapist is far less, leading to disuse of such markers at the time of admission for rehabilitation. Secondly, statistical tests of significance and regression modeling are likely to provide inaccurate inferences as noted by Jongboloed in his review ${ }^{9}$.

There has been a notable improvement in identification and modification of cardiovascular risk factors over the past decades. Better health care, public awareness, and improved socio-economic status among others have continued to increase life expectancy globally. We believe these changes have led to the observation of increase in number of stroke in the elderly (60 y and above) and the very elderly (over $80 \mathrm{y}$ ) with diminishing incidence of stroke in the younger population. Mean age of $70.7 \mathrm{y}$ as noted in our cohort of patients was uncommon in studies that were highlighted earlier in our discussion. Presence of chronic diseases has relevance towards morbidity and mortality and they are cumulative with age, thereby further differentiating our cohort from studies of younger patients.

Most of the recovery following stroke is noted over the first month and this is particularly seen in improvement of motor function ${ }^{10}$. We have recognized several other prognostic indicators for discharge destination that could be identified at the time of admission to stroke rehabilitation. These include total admission FIM score, FIM for upper limb dressing, length of stay in the acute care hospital prior to admission to stroke rehabilitation, age, and carer support.

Recovery of motor function parallels independence of daily living ${ }^{11}$. There are 13 variables routinely assessed in motor system of FIM (Table 2). All of which are given a score out of 7 (Table 3). Total FIM also include scores for communication and social cognition. It is conceivable that a positive correlation is notable in relation to higher total FIM vs returning home. To our knowledge our study is the only one that had analysed individual variables of admission motor FIM in relation to outcome performance. Of particular interest is our finding of FIM of the upper body dressing less than 5 being associated to poor functional outcome resulting in discharge to high level of care nursing home. There has been others who have demonstrated the importance of motor function over cognition in relation to functional independence ${ }^{11}$ but the importance of upper body dressing over other motor aspects warrants further analysis to clarify the role of upper limb rehabilitation. Patient recruitment to rehabilitation programs across Australia is heterogeneous in nature. Our policy required patient selection by a Geriatrician at the acute care hospital. Delay in recruitment often is a result of patient requiring medical management of active medical conditions such as febrile illness, marked biochemical abnormalities or other acute medical conditions. This reflects our finding of length of stay at the acute care hospital $14 \mathrm{~d}$ or over as a poor prognostic indicator. Therefor, extrapolation of length of stay at the acute care hospital should be carried out with caution. The impact of advancing age as a poor prognostic indicator is seen across all aspects of medical outcomes. Reduced regenerative capacity and cumulative effects of chronic diseases including degenerative disorders in advancing age are some of possible causes influencing poor prognosis in our cohort of ageing patients and is also noted in literature ${ }^{9}$. Presence of carer support has repeatedly shown in literature to be a determinant of discharge destination and is the most consistent variable analysed between studies. We believe further studies taking comorbidities into consideration would improve the predictive value of variables analysed. We plan to use our predictive variables in a prospective study to assess their sensitivity and specificity with a view to developing a scoring system. 
Table 2. Components of Functional Impairment Measurement Score (FIM)

\begin{tabular}{ll}
\hline Motor System & Cognitive System \\
\hline Feeding & Comprehension \\
Grooming & $\begin{array}{l}\text { Expression } \\
\text { Bathing }\end{array}$ \\
Dressing - upper body & Problem Solving \\
Dressing - lower body & Memory \\
Toileting & \\
Bladder management & \\
Bowel management & \\
Bed, Chair, Wheelchair transfer & \\
Toilet transfer & \\
Tub, Shower transfer & \\
Walk/Wheelchair & \\
Stairs & \\
\hline
\end{tabular}

Table 3. FIM scoring criteria. Scores 5 to 3 require a helper (modified dependence). Scores below 3 require complete dependence

Complete independence

Modified independence (patient requires use of a device, but no physical assistance)

Supervision or setup

Minimal contact assistance (patient can perform $75 \%$ or more task)

Moderate assistance (patient can perform $50 \%$ to $74 \%$ of task)

Maximum assistance (patient can perform $25 \%$ to $49 \%$ of task)

Total assistance (patient can perform less than $25 \%$ of the task or requires more than one person to assist)

\section{Conclusion}

Use of total FIM score together with other predictors prior to enrolling in inpatient stroke rehabilitation would improve planning of rehabilitation programs, predicting outcome, efficiency of rehabilitation programs and patient outcomes.

\section{References}

1. Wolfe CD. The impact of stroke. Br Med Bull 2000; 56 : 275-86.

2. Murray CJ, Lopez AD. Evidence-based health policy-lessons from the Global Burden of Disease Study. Science 1996; 274: 740-3.

3. Thomas T, Stephen B, Colin M. The global burden of cerebrovascular disease. In Cerebovascular disease. pp. 67. Geneva: WHO; 2000: 67.

4. Lozano R, Naghavi M, Foreman K, Lim S, Shibuya K, Aboyans V, Abraham J, Adair T, Aggarwal R, Ahn SY, et al. Global and regional mortality from 235 causes of death for 20 age groups in 1990 and 2010: a systematic analysis for the Global Burden of Disease Study 2010. Lancet 2012; 380: 2095-128.

5. How we manage stroke in Australia [http://www.aihw. gov.au/publication-detail/?id=6442467815].

6. Kohler F, Renton R, Dickson HG, Estell J, Connolly CE. Subacute casemix classification for stroke rehabilitation in Australia. How well does AN-SNAP v2 explain variance in outcomes? Aust Health Rev 2011; 35: 1-8.

7. Kitsos G, Harris D, Pollack M, Hubbard IJ. Assessments in Australian stroke rehabilitation units: a systematic review of the post-stroke validity of the most frequently used. Disabil Rehabil 2011; 33: 2620-32.

8. Cadilhac DA, Ibrahim J, Pearce DC, Ogden KJ, McNeill J, Davis SM, Donnan GA, Group SS. Multicenter comparison of processes of care between Stroke Units and conventional care wards in Australia. Stroke 2004; 35:1035-40.

9. Jongbloed L. Prediction of function after stroke: a critical review. Stroke 1986; 17: 765-76.

10. Duncan PW, Goldstein LB, Matchar D, Divine GW, Feussner J. Measurement of motor recovery after stroke. Outcome assessment and sample size requirements. Stroke 1992; 23: 1084-9.

11. Lincoln NB, Blackburn M, Ellis S, Jackson J, Edmans JA, Nouri FM, Walrer MF, Haworth $\mathrm{H}$. An investigation of factors affecting progress of patients on a stroke unit. J Neurol Neurosurg Psychiatry 1989; 52: 493-6. 